\title{
Fifty Years of CBMS
Regional Conferences
}

David M. Bressoud

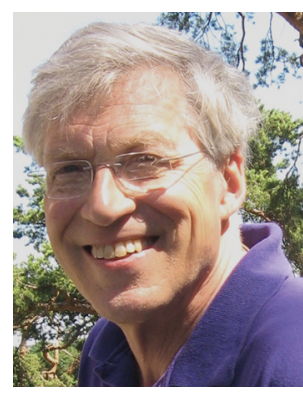

David M. Bressoud
The year 2019 marks the 50th anniversary of the CBMS/ NSF Regional Conference Series ${ }^{1}$ and the accompanying CBMS monographs https: //bit . 1y/2nZHqdN, one of the hallmark efforts of the Conference Board of the Mathematical Sciences. Since its inception in 1969, 372 of these conferences have been run, leading to the publication of more than 230 monographs. This is an opportune moment to look back on the success of this program and to look forward. While the essence of these conferences will not change, CBMS and NSF have discussed modifications that will be in place by 2020 .

\section{Origins}

CBMS was incorporated in 1960 as a forum for bringing together the presidents of the professional societies in the mathematical sciences to coordinate their efforts. Toward the end of that first decade it formulated two projects that spanned the interests of multiple societies: the departmental survey, which has been conducted every five years, and the Regional Conference Series, originally run under the auspices of both AMS and the Society for Industrial and Applied Mathematics (SIAM).

To distinguish them from the many other mathematical conferences run each year, the Regional Conferences were conceived with three distinctive features:

1. They would be regional, spread across the United States and favoring institutions that would normally not host major research conferences. The intent was to facilitate

David Bressoud is DeWitt Wallace Professor of Mathematics at Macalester College and Director of the Conference Board of the Mathematical Sciences. His email address is Bressoud@macalester . edu.

${ }^{1}$ https://www.cbmsweb.org/regional-conferences/. The work of CBMS on the Regional Conference Series is supported by NSF grants \#1709265 and $\# 1804259$. The opinions expressed in this article do not necessarily reflect those of the National Science Foundation.

For permission to reprint this article, please contact: reprint -permission@ams .org.

DOI: https://dx.doi.org/10.1090/noti 1754 access to at least one of these conferences for all mathematical scientists and to promote the formation of local research groups.

2. They would feature one leading researcher who would give a series of ten lectures over five days, leading the participants from a basic introduction in an important area of current research to the point where they could begin to work in this field.

3. The conference would be followed by the production of a monograph, based on the ten lectures, that would serve the broader population of mathematical scientists as an introduction to an exciting realm of contemporary mathematical research.

The first two monographs, both products of 1969 conferences, were Irving Kaplansky's Algebraic and Analytic Aspects of Operator Algebras [8], published by AMS, and Garett Birkhoff's The Numerical Solution of Elliptic Equations [1], published by SIAM. In the early years, SIAM published the monographs in statistics, but starting with Morris Eaton's Group Invariance Applications in Statistics [3], based on a 1987 conference, the American Statistical Association and the Institute of Mathematical Statistics have jointly published the monographs in statistics. There are now three monograph series: the CBMS Regional Conference Series in Mathematics (AMS), the CBMS-NSF Regional Conference Series in Applied Mathematics (SIAM), and the CBMS-NSF Regional Conference Series in Probability and Statistics (IMS \& ASA).

\section{Current Status}

The program has been an enormous success. Total sales of the monographs have far exceeded a quarter of a million copies. Five have gone into second editions $[5,6,10,11,14]$, and three have sold over 10,000 copies each $[2,4,13]$. One of these, Ingrid Daubechies' Ten Lectures on Wavelets, based on her 1990 CBMS Conference at the University of Lowell, has sold over 16,000 copies, been cited almost 30,000 
times, and, in 1994, received the AMS Steele Prize for Excellence in Expository Writing.

CBMS has always managed the infrastructure for these seminars: encouraging applications to NSF to run one of these conferences and disseminating information on the application process, publicizing the conferences, handling the honoraria, encouraging speakers to turn in the monographs, and approving the monographs for publication.

The CBMS lecturers have included four Fields Medalists. Paul Cohen at Carleton College in 1972 and William Thurston at the University of Minnesota in 1983 gave their lectures after receiving the medal. Vaughan Jones at the U.S. Naval Academy in 1988 and Terrence Tao at New Mexico State University in 2005 lectured before they received the medal. Women have been underrepresented as conference lecturers, but the past five years have included Wen-Ching Winnie Li [9], Nalini Joshi, Alice Guionnet, and Anna C. Gilbert among the CBMS lecturers.

Geographic, institutional, and subject diversity continue to be distinguishing features of the conference series. In the past five years regional conferences have been held in Alabama, California, Illinois, Iowa, Maryland, Minnesota, Montana, New Hampshire, New Mexico, New York, North Carolina, North Dakota, Ohio, Oklahoma, South Carolina, Texas, Utah, Washington DC, and Wyoming. They have been run at Historically Black Colleges and Universities and Hispanic Serving Institutions, at liberal arts colleges, at regional comprehensive universities, and at research universities. Topics have ranged from combinatorics, number theory, geometry/topology, algebra, and analysis to PDEs, data analysis, statistical analysis, computational methods, and quantum field theory, often focusing on exciting new developments where these subjects overlap.

In the early days, CBMS and its advisory board received a block grant from NSF, with total control over which con-

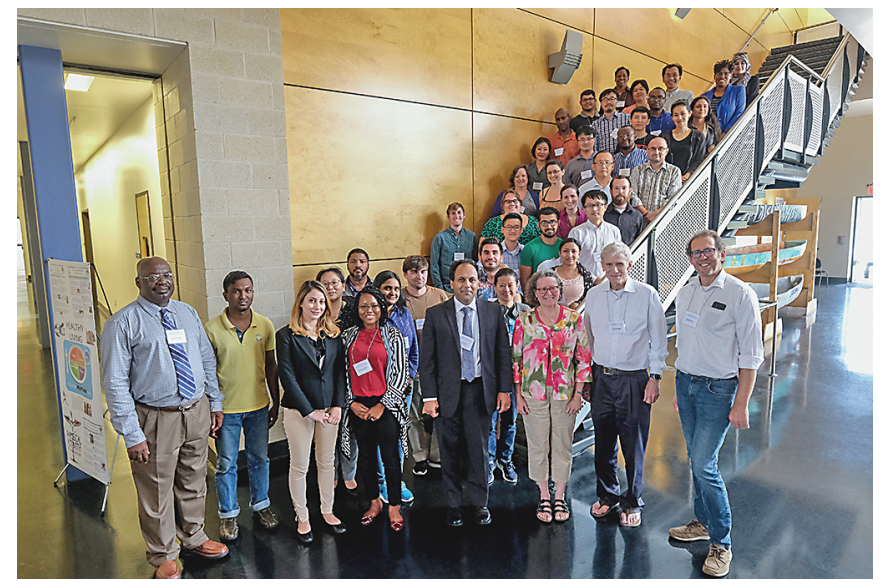

Participants at the CBMS-NSF Conference on Computational Methods in Optimal Control by William Hager at Jackson State University, July 23-27, 2018, funded by NSF grant \#1743826. ferences would be supported and at what level of funding. Over the decades, the funding model has changed. Applications to organize a CBMS conference are now made directly to NSF, usually with an April deadline to run a conference in the summer of the following year. Until recently, CBMS played a role in deciding which conferences would be funded. Moving forward, CBMS will play a more active role in soliciting applications and proposal development, and will not participate in funding decisions.

\section{Evaluating the regional conference series}

Early in 2017, in response to concerns that the number of proposals to run one of these conferences had been in decline in recent years, that monographs were coming from an ever smaller fraction of conference speakers, and that the citation numbers for recently produced monographs were also down, CBMS undertook a thorough re-evaluation of the program.

In spring and summer 2017 the board held focus groups and sent questionnaires to many of those who had been involved with CBMS conferences over the past fifteen years. The issues targeted for discussion included whether CBMS should continue to build the conferences around a single speaker giving ten lectures, whether to continue to produce monographs, and how to take advantage of the opportunities afforded by the internet. While responses varied greatly, there were three clear messages that came through:

1. A single lecturer is neither necessary nor always desirable. However, there was consistent support for the rationale behind the requirement of a single speaker: to provide a well-connected and tightly focused flow that moves from a basic introduction to the topic up to the edge of current research, giving participants an intensive experience that effectively introduces them to the important tools and problems. Many mathematicians felt that this could be accomplished more effectively with more than one voice, provided there was tight coordination in linking the talks.

2. There was almost universal support for continuation of the monographs. This came from senior mathematicians who treasure their dog-eared copies of past monographs, but also from current graduate students who continue to find them to be a useful guide into a field they wish to study. It was gratifying to see that print still has an important role to play in disseminating mathematical knowledge.

3. There also was strong support for insisting that all conferences produce a body of timely online material, from background material that should be posted before the conference begins to videos of the conference talks and detailed notes that expand on the actual talks.

We also heard of the importance of the networking that happens at these conferences and the need for opportunities to work collectively on problems and to gain experience with the software that is often an integral part of particular research streams. 
Ultimately, it was clear that CBMS, through its regional conferences, is uniquely situated to facilitate the placement of research conferences at institutions and in locations where they would not normally be found, thus helping to build the community of those actively engaged in mathematical research.

\section{Changes to the regional conference series}

Going forward, in response to community and stakeholder feedback, CBMS has implemented some changes in the conference series:

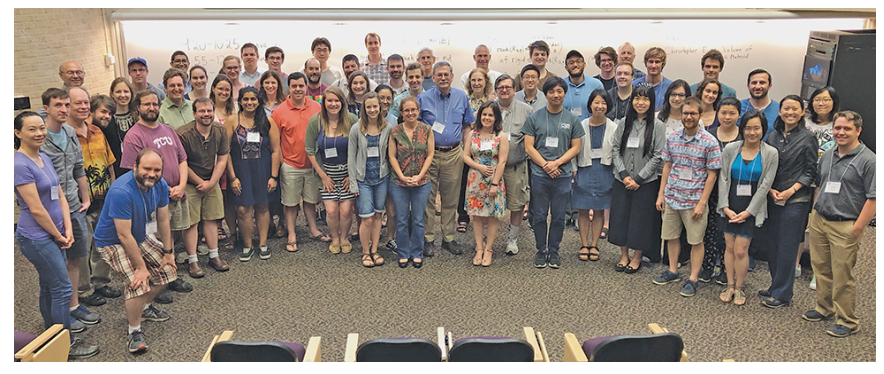

Participants at the CBMS-NSF Conference on Applications of Polynomial Systems by David Cox at Texas Christian University, June 4-8, 2018, funded by NSF grant \#1741730.

- CBMS will take a more active role in identifying both potential lecturers and potential conference sites. To assist in this effort, an Advisory Board has been constituted whose initial members are Susmita Datta, University of Florida; David Donoho, Stanford University; Bengt Fornberg, University of Colorado, Boulder; Ken Ono, Emory University; and Mary Lou Zeeman, Bowdoin College. In addition, CBMS will offer assistance in crafting strong proposals.

- The format of ten lectures over five days will be maintained, but these could be given by as many as three people provided there is only one "principal lecturer" who plans and coordinates the talks so that they stay tightly focused and build from a basic introduction to questions of current research.

- In addition, while lectures provide an efficient means of communicating a lot of information in a short space of time, by themselves they are not sufficient to engender the kind of research activity this series intends to promote. Participants, especially those new to the field, must be nurtured through interaction with others at the conference. Facilitating this interaction is encouraged, whether through problem sessions, laboratory experiences, small group Q\&A and discussions, or other means.

- There will be new expectations for materials to be posted on the CBMS website:
- The principal lecturer will provide preliminary materials that will include an introduction to the problems to be discussed. This will supply some historical context, a reading list, basic definitions and results relevant to the upcoming conference, as well as an outline of the ten talks to be given.

- The principal lectures will use prepared slides (e.g. PDF, PPT, or Beamer), which will be submitted to CBMS by the end of the conference.

- The conference organizers will video record at least the ten principal lectures, which CBMS will offer on Vimeo or a comparable site.

- The principal lecturer will post a fleshed out version of the outline of the ten talks within 45 days of the conclusion of the conference.

- A polished monograph is still expected. It is hoped that the extended outline of the talks will be a significant step toward its production.

- The hallmark emphasis on institutional and geographic diversity will be expanded to include diversity of lecturers and participants, with an emphasis on broadening participation by members of groups underrepresented in the mathematical sciences.

When, in 2017, CBMS contacted those who had recently been involved with this series, we were heartened by the outpouring of support that we received. While not everyone agreed on the way forward, everyone communicated the value of this series, both in the past and for the future. We are grateful for the legacy of the past fifty years and for this opportunity to re-imagine this series as we move into the next decades.

\section{References}

[1] Birkhoff G. The numerical solution of elliptic equations, CBMS-NSF Regional Conference Series in Applied Mathematics, 1, Published for the Conference Board of the Mathematical Sciences, Washington, DC; by the Society for Industrial and Applied Mathematics, Philadelphia, PA, 1972, MR0311125

[2] Daubechies I. Ten lectures on wavelets, CBMS-NSF Regional Conference Series in Applied Mathematics, 61, Published for the Conference Board of the Mathematical Sciences, Washington, DC; by the Society for Industrial and Applied Mathematics, Philadelphia, PA, 1992, MR1162107

[3] Eaton ML. Group Invariance Applications in Statistics, CBMS-NSF Regional Conference Series in Probability and Statistics, 1, Published for the Conference Board of the Mathematical Sciences, Washington, DC; by the Institute of Mathematical Statistics, Hayward, CA and the American Statistical Association, Alexandria, VA, 1989

[4] Efron B. The jackknife, the bootstrap, and other resampling plans, CBMS-NSF Regional Conference Series in Applied Mathematics, 38, Published for the Conference Board of the Mathematical Sciences, Washington, DC; by the Society for Industrial and Applied Mathematics, Philadelphia, PA, 1982, MR0659849

[5] Graham R, Butler S. Rudiments of Ramsey theory: Second edition, CBMS Regional Conference Series in Mathematics, 
123. Published for the Conference Board of the Mathematical Sciences, Washington, DC; by the American Mathematical Society, Providence, RI, 2015. MR340921

[6] Huber PJ. Robust Statistical Procedures: Second edition, CBMS-NSF Regional Conference Series in Applied Mathematics, 68, Published for the Conference Board of the Mathematical Sciences, Washington, DC; by the Society for Industrial and Applied Mathematics, Philadelphia, PA, 1996, MR1420192

[7] Jones VFR. Subfactors and Knots, CBMS Regional Conference Series in Mathematics, 80, Published for the Conference Board of the Mathematical Sciences, Washington, DC; by the American Mathematical Society, Providence, RI, 1991. MR1134131

[8] Kaplansky I. Algebraic and Analytic Aspects of Operator Algebras, CBMS Regional Conference Series in Mathematics, 1, Published for the Conference Board of the Mathematical Sciences, Washington, DC; by the American Mathematical Society, Providence, RI, 1970. MR0312283

[9] Li W. Zeta and L-functions in number theory and combinatorics, CBMS Regional Conference Series in Mathematics, Published for the Conference Board of the Mathematical Sciences, Saint Paul, MN; by the American Mathematical Society, Providence, RI, In Press.

[10] Rheinboldt WC. Methods for solving systems of nonlinear equations: Second edition, CBMS-NSF Regional Conference Series in Applied Mathematics, 70, Published for the Conference Board of the Mathematical Sciences, Washington, DC; by the Society for Industrial and Applied Mathematics, Philadelphia, PA, 1998. MR1645489

[11] Spencer J. Ten lectures on the probabilistic method: Second edition, CBMS-NSF Regional Conference Series in Applied Mathematics, 64, Published for the Conference Board of the Mathematical Sciences, Washington, DC; by the Society for Industrial and Applied Mathematics, Philadelphia, PA, 1993, MR1249485

[12] Tao T. Nonlinear dispersive equations: Local and global analysis, CBMS Regional Conference Series in Mathematics, 106, Published for the Conference Board of the Mathematical Sciences, Washington, DC; by the American Mathematical Society, Providence, RI, 2006. MR2233925

[13] Tarjan RE. Data structures and network algorithms, CBMSNSF Regional Conference Series in Applied Mathematics, 44, Published for the Conference Board of the Mathematical Sciences, Washington, DC; by the Society for Industrial and Applied Mathematics, Philadelphia, PA, 1983, MR0826534

[14] Temam R. Navier-Stokes equations and nonlinear functional analysis: Second edition, CBMS-NSF Regional Conference Series in Applied Mathematics, 66, Published for the Conference Board of the Mathematical Sciences, Washington, DC; by the Society for Industrial and Applied Mathematics, Philadelphia, PA, 1995, MR1318914

\section{Credits}

Author photo is courtesy of Jan Bressoud.

Photo of participants at the Cox conference is courtesy of Derek Isensee.

Photo of participants at the Hager conference is courtesy of Tor A. Kwenbe.

\section{Recent volumes from MSJ}

Advanced Studies in Pure Mathematics http://mathsoc.jp/publication/ASPM/

\section{Volume 78 \\ Singularities in Generic Geometry \\ Edited by S. Izumiya (Hokkaido), \\ G. Ishikawa (Hokkaido), \\ M. Yamamoto (Hirosaki), K. Saji (Kobe), \\ T. Yamamoto (Tokyo Gakugei), \\ M. Takahashi (Muroran Inst Tech) \\ ISBN 978-4-86497-055-6}

\section{Volume 77}

The 5oth Anniversary of Gröbner Bases Edited by T. Hibi (Osaka)

ISBN 978-4-86497-052-5

Volume 76

Representation Theory, Special Functions and Painlevé Equations RIMS 2015

Edited by H. Konno (Tokyo Marine Sci. \& Tech.),

H. Sakai (Tokyo), J. Shiraishi (Tokyo), T. Suzuki (Kindai), Y. Yamada (Kobe)

ISBN 978-4-86497-050-1

\section{MSJ Memoirs}

http://mathsoc.jp/publication/memoir/memoirs-e.html

\section{Volume 36}

Global solutions and

the asymptotic

behavior for nonlinear wave equations with

small initial data

Soichiro Katayama

ISBN 978-4-86497-054-9

Volume 35

Foundations of

the minimal model program

Osamu Fujino

ISBN 978-4-86497-045-7

$$
\nabla \nabla \nabla \text { For purchase, visit } \nabla \nabla \nabla
$$

http://www.ams.org/bookstore/aspmseries http://www.worldscientific.com/series/aspm http://www.worldscibooks.com/series/msjm

The Mathematical Society of Japan 34-8, Taito 1-chome, Taito-ku

Tokyo, JAPAN

http://mathsoc.jp/en/ 